\title{
Evolutionary Clustering Algorithm with Knowledge-Based Evaluation for Fuzzy Cluster Analysis of Gene Expression Profiles
}

\author{
Han-Saem Park and Sung-Bae Cho \\ Department of Computer Science, Yonsei University, \\ Biometrics Engineering Research Center, \\ 134 Shinchon-dong, Sudaemoon-ku, Seoul 120-749, Korea \\ sammy@sclab.yonsei.ac.kr, sbcho@cs.yonsei.ac.kr
}

\begin{abstract}
Clustering method, which groups thousands of genes by their similarities of expression levels, has been used for identifying unknown functions of genes. Fuzzy clustering method that is one category of clustering assigns one sample to multiple groups according to their membership degrees. It is more appropriate than hard clustering algorithms for analyzing gene expression profiles since single gene might involve multiple genetic functions. However, general clustering methods have problems that they are sensitive to initialization and can be trapped into local optima. To solve the problems, we propose an evolutionary fuzzy clustering algorithm with knowledge-based evaluation. It uses a genetic algorithm for clustering and prior knowledge of data for evaluation. Yeast cell-cycle dataset has been used for experiments to show the usefulness of the proposed method.
\end{abstract}

\section{Evolutionary Fuzzy Clustering with Knowledge-Based Evaluation}

General clustering algorithms have common problems that they are very sensitive to initial values and they can be trapped by local optima since their processes are supposed to minimize objective function [1]. Besides, there is a problem of evaluating cluster results. Since gene expression profiles vary depending on their characteristics and environments that they were collected, it is not appropriate to evaluate them with the same criteria.

We propose an evolutionary fuzzy clustering and knowledge-based evaluation method to solve the problems. GA (genetic algorithm) that is an efficient method to solve optimization problem is applied for the evolutionary fuzzy clustering method. There have been many publications related to evolutionary computation for clustering. Maulik and Bandyopadhyay tried to minimize the distances between the data in the same clusters and cluster centers [1], and Hall used GA to minimize objective function value of the hard and fuzzy c-means algorithms. However, they fixed the number of clusters and used GA only for the minimization of objective function. We have encoded one cluster partition of variable number of clusters as one chromosome and formed various cluster partitions. 
The proposed method is divided into two parts: an evolutionary clustering part, which searches optimal cluster partition using GA, and a knowledge-based evaluation part, which obtains the optimal $\alpha$-cuts from several datasets for Bayesian validation (BV) method. Fuzzy c-means algorithm known as the most widely used fuzzy clustering method is used for clustering [2].

For knowledge-based evaluation, we have used BV and decision tree (DT) rule to decide the optimal $\alpha$-cut value. Original BV [3] evaluates cluster partition with the same $\alpha$-cuts for all datasets, but it cannot evaluate the cluster results correctly since each dataset has different distribution and they are extracted from different environments.

We have obtained $\alpha$-cut value for each dataset using the DT rule. First, $N$ gene expression profiles are clustered using the fuzzy c-means algorithm, and the results are evaluated by BV. Subsequently, the optimal $\alpha$-cut for each dataset is decided, and they are used for the labels of DT training data. Rule production process trains DT, and produces rules. As Fig. 1 illustrates, the attributes of DT training data are produced using membership matrices that are the fuzzy clustering results of each dataset. Incrementing the membership degree value from 0.0 to 1.0 with the difference of 0.1 , attributes are divided into 10 sections. Each section counts the frequency of samples and calculates the attribute by dividing the frequency by the total number of samples. These attributes calculated are $A_{1} \sim A_{10}$.

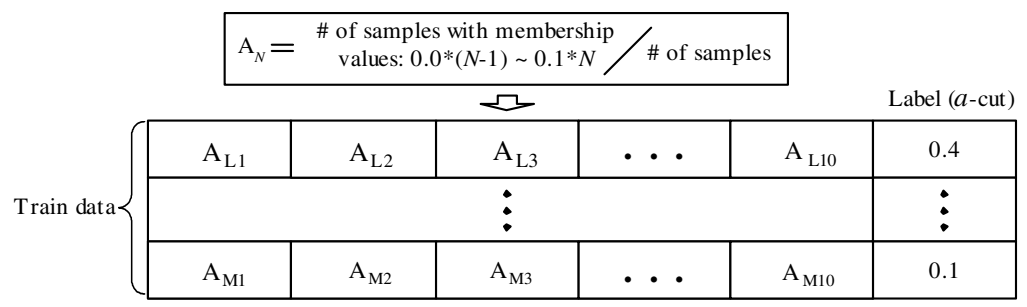

Fig. 1. Training data production process of decision tree

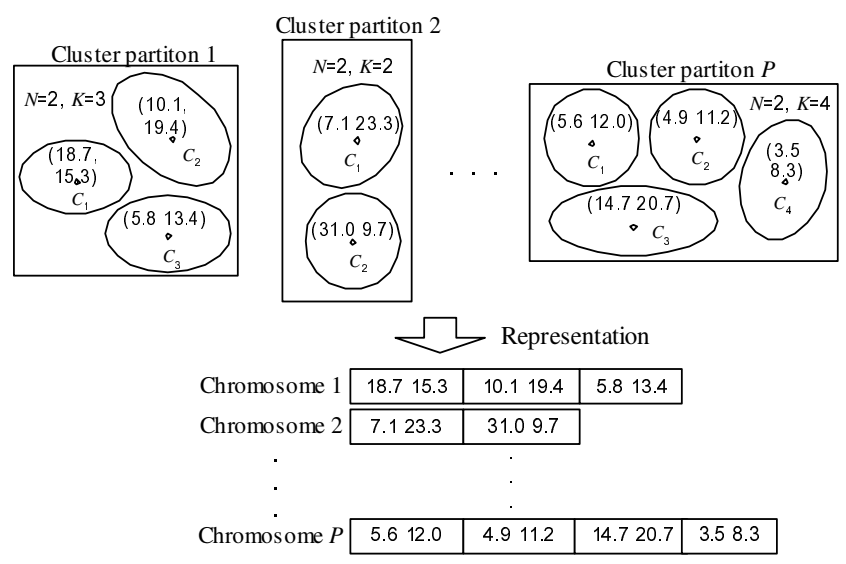

Fig. 2. Representation of variable length chromosome 
The training process sets $\alpha$-cut value when experimental dataset is inputted. Using these $\alpha$-cut values, BV calculates the final Bayesian score (BS), which is the degree of fitness evaluation.

For evolutionary clustering, we have used floating point representation to represent a set of cluster centers of cluster partition. One cluster partition contains $K$ clusters, and a chromosome is represented in a space of $N \times K$ in case that the dimension of each center is $N$. Fig. 2 illustrates several chromosomes that are in one cluster partition, and each chromosome can have different number of clusters and different values of cluster centers.

Population is initialized at random. The cluster numbers less than square value of the sample size have been used for clustering. The minimum number of clusters is set as 2. To evaluate fitness, all samples are clustered using fuzzy c-means algorithm, first. After cluster centers are updated, the chromosomes with updated centers are evaluated by BV. A roulette wheel strategy is used for selection. Crossover and mutation operations are performed considering variable length chromosomes.

\section{Experiments and Analyses}

We have performed experiments with yeast cell-cycle dataset, and it has expression levels of 6000 genes expressed during two cell cycles. 421 genes that show significant change of expression levels are used here, referring related work [4].

Maximum generation number of 1000 and population size of 200 have been used for experiments. Maximum numbers of clusters have been set by 20, and crossover rate of 0.8 and mutation rate of 0.01 have been used. The fuzziness parameter of the fuzzy c-means algorithm has been set as 1.2.

$\alpha$-cut value of yeast cell-cycle dataset is decided as 0.4 by DT. Table 1 shows the training data extracted from yeast cell-cycle and SRBCT datasets. Comparing SRBCT, Values in $\mathrm{A}_{2}$ and $\mathrm{A}_{9}$ of yeast cell-cycle dataset are larger, and the value in $\mathrm{A}_{10}$ is smaller because the samples of yeast cell-cycle dataset have fuzzier cluster boundaries than SRBCT dataset. This shows that the characteristics of dataset should be considered since they are different.

Table 1. Training data of DT

\begin{tabular}{ccccccccccc}
\hline & \multicolumn{10}{c}{ Attributes of training data } \\
\cline { 2 - 11 } Dataset & $\mathrm{A}_{1}$ & $\mathrm{~A}_{2}$ & $\mathrm{~A}_{3}$ & $\mathrm{~A}_{4}$ & $\mathrm{~A}_{5}$ & $\mathrm{~A}_{6}$ & $\mathrm{~A}_{7}$ & $\mathrm{~A}_{8}$ & $\mathrm{~A}_{9}$ & $\mathrm{~A}_{10}$ \\
\hline Yeast & 1.000 & 0.231 & 0.114 & 0.086 & 0.071 & 0.064 & 0.059 & 0.069 & 0.162 & 0.546 \\
SRBCT & 1.000 & 0.016 & 0.000 & 0.016 & 0.000 & 0.016 & 0.000 & 0.000 & 0.048 & 0.937 \\
\hline
\end{tabular}

We have compared the proposed method with the original FCM by means of BS and the objective function value (OF value) of the FCM. Table 2 shows 10 experimental results of yeast cell-cycle dataset. If $\mathrm{BS}$ is high and the objective function value is low, it means that clustering is performed well since the objective function value is based on the distances between cluster centers and samples. The proposed method shows better results than original FCM in both BS and OF value. 
Table 2. Comparison experiments of original FCM and GA+FCM

\begin{tabular}{ccccc}
\hline & \multicolumn{2}{c}{ Original FCM } & \multicolumn{2}{c}{ GA+FCM } \\
\cline { 2 - 5 } Count & BS & OF value & BS & OF value \\
\hline $\mathbf{1}$ & 0.03354 & 164.472 & 0.13256 & 166.883 \\
$\mathbf{2}$ & 0.00875 & 163.670 & 0.11246 & 161.542 \\
$\mathbf{3}$ & 0.03238 & 165.057 & 0.12661 & 162.911 \\
$\mathbf{4}$ & 0.03825 & 162.653 & 0.08058 & 162.073 \\
$\mathbf{5}$ & 0.02165 & 163.758 & 0.10667 & 162.798 \\
$\mathbf{6}$ & 0.04096 & 164.086 & 0.09778 & 162.312 \\
$\mathbf{7}$ & 0.02806 & 163.052 & 0.11873 & 162.042 \\
$\mathbf{8}$ & 0.04473 & 164.877 & 0.13659 & 162.773 \\
$\mathbf{9}$ & 0.02478 & 162.452 & 0.12898 & 162.905 \\
$\mathbf{1 0}$ & 0.04645 & 169.216 & 0.11246 & 161.542 \\
\hline Average & 0.03195 & 164.329 & 0.11534 & 162.778 \\
\hline
\end{tabular}

Finally, we have compared and analyzed the experimental result with the known genes of Cho's work [4]. We have focused on fuzzy genes that belong to several clusters at the same time with the membership degrees higher than 0.3. Fig. 3 illustrates the gene description and cluster number of fuzzy genes.

\begin{tabular}{|c|c|c|c|}
\hline Gene & & Gene description & Clusters \\
\hline YPR019W & & member of the $\mathrm{Cdc} 46 \mathrm{p} / \mathrm{Mcm} 2 \mathrm{p} / \mathrm{Mcm} 3 \mathrm{p}$ family & 10,3 \\
\hline YHR113W & & similarity to vacuolar aminopeptidase Ape $1 \mathrm{p}$ & 20,21 \\
\hline YHR038W & & killed in mutagen & 20,21 \\
\hline YNL078W & & hypothetical protein & $3,19,25$ \\
\hline YBR160W & & "g1,g2" CDC28 cyclin-dependent kinase & $6,12,24$ \\
\hline YDL227C & & homothallic switching endonuclease & $5,12,26$ \\
\hline YER070W & & ribonucleoside-diphosphate reductase, large subunit & $12,24,26$ \\
\hline YOL017W & & similarity to YFR013W & \\
\hline YDR464W & & regulates spliceosome components & 9,11 \\
\hline YCR086W & & hypothetical protein & 9,11 \\
\hline YKL052C & & hypothetical protein & 9,11 \\
\hline YPR111W & & kinase involved in late nuclear division & 9,13 \\
\hline YL050W & & cyclin like protein interacting with $\mathrm{Pho85p}$ & 11,13 \\
\hline YHRO23W & & myosin-1 isoform heavy chain & 7,18 \\
\hline YOR315W & & hypothetical protein & 7,18 \\
\hline
\end{tabular}

Fig. 3. Gene description and cluster number of fuzzy genes

\section{Acknowledgements}

This work was supported by the Korea Science and Engineering Foundation (KOSEF) through the Biometrics Engineering Research Center (BERC) at Yonsei University. 


\section{References}

[1] U. Maulik and S. Bandyopadhyay, "Genetic algorithm-based clustering technique," Pattern Recognition, vol. 33, pp. 1455-1465, 2000.

[2] F. Hoppner, et al., Fuzzy Cluster Analysis, Wiley, 1999.

[3] S.-H. Yoo, et al., "Analyzing fuzzy partitions of Saccharomyces cerevisiae cell-cycle gene expression data by Bayesian validation method," Proc. of the 2004 IEEE Symposium on CIBCB, pp. 116-122, 2004.

[4] R. J. Cho, et al., "A genome-wide transcriptional analysis of the mitotic cell cycle," Molecular Cell, vol. 2, pp. 65-73, 1998. 